\title{
Enfermedad periodontal como factor de riesgo adicional asociado con nacimiento pretérmino en México: un estudio de casos y controles
}

\author{
J. Jesús Pérez-Molina, ${ }^{1}$ María Janeth González-Cruz, ${ }^{1}$ J. Guadalupe Panduro-Barón, ${ }^{2}$ \\ Luz Patricia Santibáñez-Escobar, ${ }^{3}$ Norma Argelia Quezada-Figueroa ${ }^{4}$ y Martín Bedolla-Barajas ${ }^{5}$
}

${ }^{1}$ Hospital Civil de Guadalajara "Dr. Juan I. Menchaca", División de Pediatría, Servicio de Neonatología; ${ }^{2}$ Hospital Civil de Guadalajara "Dr. Juan I. Menchaca", División de Ginecología y Obstetricia; ${ }^{3}$ Hospital Civil de Guadalajara "Dr. Juan I. Menchaca", Servicio de Cirugía Maxilofacial; "Universidad de Guadalajara, Centro Universitario de Ciencias de la Salud, Departamento de Clínicas de la Reproducción Humana, Crecimiento y Desarrollo Infantil; ${ }^{5}$ Hospital Civil de Guadalajara "Dr. Juan I. Menchaca", Servicio de Alergia e Inmunología Clínica. Guadalajara, Jalisco, México

\section{Resumen}

Introducción: Las sustancias relacionadas con los microorganismos involucrados en la enfermedad periodontal puedan llegar a la interfaz materno-fetal por vía hematógena y estimular la contractilidad uterina. Objetivo: Determinar la asociación entre enfermedad periodontal con nacimiento pretérmino. Método: Estudio de casos y controles de 343 embarazadas pretérmino y 686 de término. Se calculó la edad gestacional por fecha de último periodo menstrual y se confirmó con los métodos de Capurro y Ballard. La enfermedad periodontal se diagnosticó por la profundidad del espacio entre la raíz dental y la encía. La asociación fue medida con regresión logística. Resultados: La edad de las madres en los casos fue de $23.8 \pm 6.7$ años y en los controles de $23.2 \pm 6.7$ años. La enfermedad periodontal estuvo presente en $66.8 \%$ de los casos y $40.5 \%$ de los controles. Los factores asociados con nacimiento pretérmino fueron enfermedad periodontal ( $R M=2.26)$, antecedente de nacimiento pretérmino $(R M=4.96)$, embarazo no planeado $(R M=2.15)$, control prenatal deficiente $(R M=2.53)$, infección de vías urinarias $(R M=2.22)$, preeclampsia $(R M=4.49)$, ruptura prematura de membranas amnióticas $(R M=2.59)$ y nacer por cesárea $(R M=9.15)$. Conclusión: La enfermedad periodontal en el embarazo constituyó un factor de riesgo independiente para nacimiento pretérmino.

PALABRAS CLAVE: Nacimiento pretérmino. Enfermedad periodontal. Factores de riesgo.

\begin{abstract}
Introduction: Substances related to microorganisms involved in periodontal disease can reach the maternal-fetal interface via the hematogenous route and stimulate uterine contractility. Objective: To determine the association between periodontal disease and preterm birth. Method: Case-control study in 343 preterm and 686 full-term pregnant women. Gestational age was calculated based on the date of the last menstrual period and confirmed with Capurro and Ballard methods. Periodontal disease was diagnosed according to the depth of the space between the tooth root and the gum. The association was measured with logistic regression. Results: Maternal age of the cases was $23.8 \pm 6.7$ years, and $23.2 \pm 6.7$ in the controls. Periodontal disease was present in $66.8 \%$ of cases and $40.5 \%$ of controls. The factors associated with preterm birth were periodontal disease (Odds ratio $[O R]=2.26$ ), history of preterm birth $(O R=4.96)$, unplanned pregnancy $(O R=2.15)$ poor prenatal control $(O R=2.53)$, urinary tract infection $(O R=2.22)$, preeclampsia $(O R=4.49)$, premature rupture of membranes $(O R=2.59)$ and caesarean section delivery $(O R=9.15)$. Conclusion: Periodontal disease in pregnancy was an independent risk factor for preterm birth.
\end{abstract}

KEY WORDS: Preterm birth. Periodontal disease. Risk factors.

Fecha de recepción: 05-04-2018

Fecha de aceptación: 06-12-2018

DOI:10.24875/GMM.18004332
Gac Med Mex. 2019;155:143-148

Disponible en PubMed

www.gacetamedicademexico.com 


\section{Introducción}

Se denomina nacimiento pretérmino (NP) al acontecido antes de 37 semanas de gestación o 259 días a partir del primer día del último periodo menstrual (FUM). ${ }^{1}$

La incidencia global del NP es de alrededor de 15 millones de NP por año y en Estados Unidos es la principal causa de morbilidad y mortalidad neonatal; ${ }^{2}$ los sobrevivientes presentan aproximadamente la mitad de los trastornos del neurodesarrollo que ocurren en la infancia, ${ }^{1}$ y los costos sociales resultantes de los gastos médicos, educacionales y por pérdida de productividad ascienden a 26200 millones de dólares anuales. ${ }^{2}$

En Estados Unidos, la tasa de NP es 12 a $13 \%$ y en Europa y otros países desarrollados la frecuencia ha sido estimada en 5 a $9 \% .^{3}$ En un estudio realizado en el Hospital Civil de Guadalajara "Dr. Juan I. Menchaca", Guadalajara, Jalisco, donde se llevó a cabo la investigación la tasa de NP fue de $6.9 \%{ }^{4}$

La labor pretérmino es un síndrome que puede ser desencadenado por múltiples mecanismos, entre los que se encuentran infecciones intrauterinas y distantes al útero, inflamación, isquemia, hemorragia uteroplacentaria, sobredistensión uterina, estrés y procesos inmunológicos. Diversas características maternas también han sido asociadas con la mayor frecuencia, entre ellas variables demográficas, nutricionales y psicológicas. ${ }^{3}$

La enfermedad periodontal (EP) es una condición inflamatoria crónica que involucra los tejidos blandos y duros que rodean al diente, así como el ligamento periodontal, el hueso y el cemento, debido a microorganismos en su mayoría gramnegativos y anaerobios, y a las sustancias relacionadas con ellos como citocinas proinflamatorias y lipopolisacáridos, que puedan llegar a la interfaz materno-fetal por vía hematógena y estimular la contractilidad uterina. 5,6

Existen estudios que han encontrado asociación entre EP y NP, ${ }^{6-10} \sin$ embargo, otros no la han documentado. ${ }^{11-13}$

En cada región geográfica pueden existir factores locales con capacidad para modificar la relación entre la EP con la frecuencia de NP, por lo que el objetivo de esta investigación fue determinar dicha asociación en un hospital público localizado en el occidente de México.

\section{Método}

Se realizó un estudio de casos y controles. ${ }^{14}$ La población de estudio fueron las díadas madre-hijo atendidas en el Hospital Civil de Guadalajara "Dr. Juan I. Menchaca", que proporciona servicios de salud a población urbana, abierta y de escasos recursos económicos de la zona metropolitana de Guadalajara, en el occidente de México, de mayo de 2011 a junio de 2012.

La muestra se constituyó con todas las díadas madre-hijo vivo de 24 a 36 semanas de gestación (SDG), incluidas consecutivamente (casos); y dos díadas madre-hijo vivo de 37 a 41 SDG por cada caso (controles); las últimas fueron seleccionadas de forma aleatoria simple entre la población de nacimientos de término.

El tamaño de la muestra se calculó con error alfa de $0.05 \%$, error beta de $20 \%$ y razón de dos controles por cada caso. Para incluir la frecuencia de exposición a EP y las posibles variables confusoras con menor diferencial de exposición entre casos y controles se consideró la variable atención prenatal deficiente, ${ }^{6}$ que en los NP fue de $11 \%$ y en los nacimientos de término de $3 \%$, a lo que se añadió $20 \%$ por posibles exclusiones; así, el cálculo sugirió 154 casos y 308 controles.

La variable dependiente fue NP (24-36 SDG) y la independiente EP. Se asumió que la EP estuvo presente cuando después de un examen bucal completo, cuatro o más dientes tuvieron al menos un sitio con profundidad $\geq 4 \mathrm{~mm}$ y pérdida clínica de tejido óseo o blando $\geq 3 \mathrm{~mm},{ }^{15}$ medidas con sonda periodontal descartable PerioWise ${ }^{\circledR}$ (Premier). La intensidad de la EP se midió de la siguiente manera:

- Grado leve, cuando cuatro o más dientes tuvieron al menos un sitio con probada profundidad $\geq 4 \mathrm{~mm}$ y al menos un diente con pérdida de unión $\geq 3 \mathrm{~mm}$ (pérdida de tejido óseo o blando).

- Grado moderado, cuando cuatro o más dientes tuvieron al menos un sitio con probada profundi$\mathrm{dad} \geq 4 \mathrm{~mm}$ y al menos un diente con pérdida de unión $\geq 4 \mathrm{~mm}$ (pérdida de tejido óseo 0 blando).

- Grado severo, cuando cuatro o más dientes tuvieron al menos un sitio con probada profundidad $\geq 4 \mathrm{~mm}$ y al menos un diente con pérdida de unión $\geq 5 \mathrm{~mm}$ (pérdida de tejido óseo o blando). ${ }^{15}$

La edad gestacional fue calculada con los días transcurridos a partir de la FUM, obtenida por entrevista directa. Cuando los neonatos tuvieron $>29$ SDG, la edad gestacional de los recién nacidos (RN) se valoró con el método de Capurro ${ }^{16}$ y en los RN $\leq 29$ SDG con la prueba de Ballard. ${ }^{17}$ Cuando hubo diferencia mayor de dos semanas entre la edad 
gestacional calculada por FUM y la medida por Capurro o Ballard y cuando la madre no recordó la FUM, se tomó como definitiva la edad gestacional obtenida por Capurro o Ballard.

Para medir las variables independiente y dependiente, uno de los investigadores se entrenó y estandarizó hasta obtener concordancia casi perfecta en dos observadores: ${ }^{18}$ para diagnosticar EP como presente 0 ausente se estandarizó con un odontólogo certificado (coeficiente kappa $=0.927$ ) para mediciones $\pm 1 \mathrm{~mm}$ entre cada par de examinadores; para cuantificar las SDG y diagnosticar NP como presente o ausente se estandarizó con un neonatólogo certificado para realizar las evaluaciones de Capurro (coeficiente kappa $=0.940$ ) y Ballard (coeficiente kappa $=0.934)$.

Los datos de la investigación se recolectaron en forma prolectiva; además de medir la variable independiente y dependiente, también se obtuvo información de primera mano por entrevistas directas a las madres en las primeras 24 horas del nacimiento del $\mathrm{RN}$, acerca de la edad, FUM, antecedente de nacimiento pretérmino no debido a indicaciones médicas, atención prenatal, estado socioeconómico, datos demográficos, embarazo planificado, uso de drogas, parejas sexuales, abuso sexual y enfermedades durante el embarazo.

Del expediente clínico, el mismo día de la entrevista se recabó información relacionada con el modo de nacimiento, ruptura espontánea de las membranas amnióticas previa al inicio del trabajo de parto, resultados de urocultivos para diagnosticar infección de vías urinarias, enfermedades durante el embarazo y datos obstétricos relevantes.

La atención prenatal fue adecuada cuando las madres recibieron como mínimo cinco consultas prenatales con el siguiente calendario: la primera en las 12 semanas iniciales, la segunda entre la 22 y 24 , la tercera entre la 27 y 29 , la cuarta entre la 33 y 35 y la quinta en la semana 36 de gestación. ${ }^{19}$ Este calendario se ajustó a la duración de la gestación en cada díada madre-hijo pretérmino.

El estado socioeconómico se valoró con la escala Esomar, ${ }^{20}$ que toma en cuenta la actividad laboral del jefe de familia, ingreso económico, educación y características de la vivienda.

Las díadas madre-hijo con información incompleta para determinar si la EP era un factor de riesgo para NP fueron eliminadas de la investigación. Se efectuó prueba piloto de recolección y captura de información antes de los registros definitivos, para detectar y corregir errores y la inconsistente no se incluyó en la base de datos definitiva.

Se calculó media y desviación estándar de las variables cuantitativas y se compararon con $t$ de Student para dos muestras independientes; las cualitativas se describieron con proporciones y compararon con $\chi^{2}$ o exacta de Fisher según fue necesario. El riesgo entre EP con NP y el efecto de confusión se midió con razón de momios (RM) por regresión logística con el método forward conditional. Se verificó el buen ajuste del modelo con la prueba de Hosmer y Lemeshow. En todos los cálculos el intervalo de confianza fue de $95 \%$ (IC $95 \%$ ). Los análisis fueron realizados con el programa estadístico SPSS versión 22.0.

La investigación fue aprobada por los comités de investigación y ética del hospital sede (registro 1101/11), las madres firmaron un consentimiento informado por escrito para participar en el estudio.

\section{Resultados}

Se obtuvo información completa en 343 díadas madre-hijo vivo pretérmino y 686 díadas madre-hijo vivo de término.

La edad media de las madres en las díadas madre-hijo pretérmino fue de $23.8 \pm 6.7$ años y en las de término $23.2 \pm 6.0$ años $(p=0.143$ ); el ingreso mensual familiar fue $4654 \pm 2016$ y $4378 \pm 2190$ pesos mexicanos $(p=0.051)$ y la escolaridad $7.9 \pm 3.0$ y $7.6 \pm 2.8$ años de estudio $(p=0.154)$, respectivamente. La edad gestacional corregida con las valoraciones de Capurro y de Ballard fue de $33.5 \pm 2.7$ semanas en el grupo de casos y de $39.1 \pm 1.1$ semanas en el grupo control. Los índices de curtosis y de asimetría de las variables mencionadas sugirieron su distribución normal.

La frecuencia de EP en el grupo de casos fue de 229/343 (66.8\%), de intensidad leve en 165/229 (72\%), moderada en $60 / 229(26.2 \%)$ y severa en $4 / 229(1.8 \%)$. En el grupo control, la frecuencia de EP fue de 278/686 (40.5\%), de intensidad leve en 268/278 (96.4\%), moderada en 10/278 (3.6 \%) y ningún caso severo.

En relación con los hábitos de salud dental durante el embarazo, 294/343 (87.7\%) mujeres de los casos y 524/686 (76.4\%) de los controles nunca tuvieron revisión dental.

En el análisis bivariado, factores como el antecedente de nacimiento pretérmino no debido a indicaciones médicas, embarazo no planificado, embarazo múltiple, control prenatal inadecuado, infección de 
Gaceta Médica de México. 2019;155

Tabla 1. Características demográficas, médicas y obstétricas de los grupos de estudio

\begin{tabular}{|c|c|c|c|c|c|c|}
\hline \multirow[t]{2}{*}{ Variables } & \multicolumn{2}{|c|}{ RN Pretérmino $n=343$} & \multicolumn{2}{|c|}{ RN Término $n=686$} & \multirow[t]{2}{*}{ RM (IC $95 \%)$} & \multirow[t]{2}{*}{$\mathrm{p}$} \\
\hline & $\mathbf{n}$ & $\%$ & $\mathbf{n}$ & $\%$ & & \\
\hline Estado civil ${ }^{\star}$ & 229 & 66.8 & 458 & 66.8 & $1(0.75-1-33)$ & 0.999 \\
\hline Ocupación ${ }^{\dagger}$ & 322 & 95.9 & 634 & 92.4 & $1.26(0.74-2.12)$ & 0.390 \\
\hline Nivel socioeconómico $\ddagger$ & 155 & 45.2 & 375 & 54.7 & $0.68(0.53-0.89)$ & 0.002 \\
\hline Tabaquismo\& & 24 & 7.0 & 51 & 7.4 & $0.94(0.55-1.59)$ & 0.799 \\
\hline Alcoholismo\& & 20 & 5.8 & 28 & 4.1 & $1.46(0.78-2.72$ & 0.209 \\
\hline Drogas ilícitas\& & 9 & 2.6 & 9 & 1.3 & $2.03(0.73-5.61)$ & 0.130 \\
\hline Parejas sexuales ${ }^{\bullet}$ & 16 & 4.7 & 24 & 3.5 & $1.35(0.67-2.69)$ & 0.361 \\
\hline Abuso sexual\& & 8 & 2.3 & 12 & 1.7 & $1.34(0.50-3.56)$ & 1.34 \\
\hline Antecedente de RNPT\& & 49 & 14.3 & 30 & 4.4 & $3.64(2.21-6.02)$ & $<0.001$ \\
\hline Embarazo no planeado\& & 119 & 34.7 & 141 & 20.5 & $2.05(1.52-2.77)$ & $<0.001$ \\
\hline Primigesta" & 129 & 37.6 & 265 & 38.6 & $0.96(0.73-1.26)$ & 0.750 \\
\hline Embarazo múltiple** & 40 & 11.7 & 5 & 0.7 & $17.98(6.71-52.3)$ & $<0.001$ \\
\hline Atención prenatal't+ & 191 & 55.7 & 283 & 41.2 & $1.79(1.37-2.35)$ & $<0.001$ \\
\hline IVU durante el embarazo\& & 82 & 23.8 & 113 & 16.5 & $1.59(1.16-2.19)$ & $<0.001$ \\
\hline Preeclampsia\& & 21 & 6.1 & 9 & 1.3 & $4.91(2.22-10.83)$ & $<0.001$ \\
\hline Diabetes mellitus\& & 8 & 2.3 & 4 & 0.6 & $4.07(1.22-13.6)$ & 0.006 \\
\hline Enfermedad periodontal ${ }^{\&}$ & 229 & 66.8 & 278 & 40.5 & $2.95(2.23-3.90)$ & $<0.001$ \\
\hline $\mathrm{RPM}^{\&}$ & 28 & 8.2 & 16 & 2.3 & $3.72(1.91-7.31)$ & $<0.001$ \\
\hline Nacimiento por cesárea ${ }^{\ddagger \ddagger}$ & 268 & 78.1 & 206 & 30.0 & $8.33(6.08-11.42)$ & $<0.001$ \\
\hline
\end{tabular}

${ }^{*}$ Casadas versus no casadas,

thogar versus otras ocupaciones.

†Nivel socioeconómico bajo y medio bajo versus medio alto y alto con la escala Esomar.

¿Sí versus no,

- $\geq$ una pareja sexual versus una pareja sexual;

"Versus > 1 embarazo;

$* * 2$ productos versus un producto;

"Inadecuada versus adecuada (NOM-007-SSA2-1993):

\#versus nacimiento vía vaginal.

$\mathrm{RN}=$ recién nacido, RPM = ruptura espontánea de las membranas amnióticas previo al inicio del trabajo de parto, RNPT = recién nacido pretérmino no debido a indicaciones médicas

IVU =: infección de vías urinarias, $\mathrm{RM}=$ razón de momios, IC = intervalo de confianza, $\mathrm{p}=$ comparación de las proporciones por chi cuadrada

vías urinarias durante el embarazo, preeclampsia, diabetes mellitus, ruptura espontánea de las membranas amnióticas previo al inicio del trabajo de parto, nacimiento por cesárea y la presencia de enfermedad periodontal fueron más frecuente en los casos $(p<0.05)$ (Tabla 1).

La frecuencia de nivel socioeconómico bajo y medio bajo fue menor en el grupo de casos y las frecuencias de tabaquismo, alcoholismo y consumo de drogas ilícitas durante el embarazo fueron semejante en los grupos de casos y controles. También el estado civil de la madre, ocupación, tener más de una pareja y el antecedente de abuso sexual se distribuyeron con frecuencia semejante en los dos grupos (Tabla 1).
Para determinar si la EP fue factor de riesgo para NP y evaluar el efecto de las covariables estudiadas, primero se excluyeron los embarazos múltiples y a través de análisis multivariados por regresión logística con el método forward conditional en los embarazos únicos. Se documentó que la enfermedad periodontal $(\mathrm{RM}=2.25, \mathrm{p}<0.001)$, el antecedente de nacimiento pretérmino no debido a indicaciones médicas, $(\mathrm{RM}=$ 4.96, $p<0.001)$, embarazo no planificado ( $R M=2.15$, $p<0.001)$, atención prenatal deficiente $(R M=2.53$, $p<0.001)$, infección de vías urinarias durante el embarazo $(\mathrm{RM}=2.22, \mathrm{p}<0.001)$, preeclampsia $(\mathrm{RM}=$ $4.49, \mathrm{p}$ 0.001), ruptura espontánea de las membranas amnióticas previo al inicio del trabajo ( $R M=2.59$, $p<0.012)$ y nacer por cesárea $(R M=9.15, p<0.001)$ 
Tabla 2. Análisis de regresión logística con nacimiento pretérmino como variable desenlace en embarazos únicos y posibles covariables confusoras

\begin{tabular}{|c|c|c|c|c|c|c|}
\hline \multirow[t]{2}{*}{ Covariables } & \multicolumn{3}{|c|}{ Análisis bivariado } & \multicolumn{3}{|c|}{ Modelo ajustado** } \\
\hline & RM & IC $95 \%$ & $\mathrm{p}$ & RM & IC $95 \%$ & $\mathrm{p}$ \\
\hline Enfermedad periodontal ${ }^{*}$ & 2.95 & $2.23-3.90$ & $<0.001$ & 2.25 & $1.61-3.14$ & $<0.001$ \\
\hline $\mathrm{RPM}^{*}$ & 3.72 & $1.91-7.31$ & $<0.001$ & 2.59 & $1.23-5.47$ & 0.012 \\
\hline Antecedente de RNPT* & 3.64 & $2.21-6.02$ & $<0.001$ & 4.96 & $2.75-8.95$ & $<0.001$ \\
\hline Embarazo no planeado* & 2.05 & $1.52-2.77$ & $<0.001$ & 2.15 & $1.49-3.12$ & $<0.001$ \\
\hline Atención prenatal ${ }^{\dagger}$ & 1.79 & $1.37-2.35$ & $<0.001$ & 2.53 & $1.80-3.56$ & $<0.001$ \\
\hline IVU durante el embarazo* & 1.59 & $1.16-2.19$ & $<0.001$ & 2.22 & $1.46-3.35$ & $<0.001$ \\
\hline Preeclampsia* & 4.91 & $2.22-10.83$ & 0.001 & 4.49 & $1.81-11.10$ & 0.001 \\
\hline Diabetes mellitus* & 4.07 & $1.22-13.8$ & 0.006 & 3.43 & $0.81-14.49$ & 0.093 \\
\hline Nacimiento por cesárea* & 8.33 & $6.08-11.42$ & $<0.001$ & 9.15 & $6.41-13.08$ & $<0.001$ \\
\hline
\end{tabular}

permanecieron como factores de riesgo para nacimiento pretérmino, mientras que desapareció la asociación con la diabetes mellitus durante el embarazo (Tabla 2).

\section{Discusión}

El presente estudio muestra que la enfermedad periodontal fue un factor de riesgo independiente de nacimiento pretérmino adicional a los factores de riesgo tradicionalmente asociados con NP, ${ }^{1-3,21}$ como antecedente de NP no debido a indicaciones médicas, atención prenatal deficiente, infección de vías urinarias durante el embarazo, preeclampsia, ruptura espontánea de las membranas amnióticas previo al inicio del trabajo y nacer por cesárea.

La asociación entre EP y NP fue sugerida por primera vez en Estados Unidos en 1996 por Ofenbacher et $a l .{ }^{6}$ en un estudio de casos y controles ( $R M=7.5$, IC $95 \%$ 1.9-28.8) y confirmada con diseños de cohortes por Jeffcoat et al. ${ }^{7}(\mathrm{RR}=4.5$, IC $95 \%=2.2-9.2)$ y Ofenbacher et al. ${ }^{8}(\mathrm{RR}=2.0$, IC $95 \%$ 1.2-3.2). Del mismo modo, con diseños de casos y controles, Siqueira et al. ${ }^{9}$ también reportaron esta asociación en Brasil (RM = 1.77; IC $95 \%$ 1.12-2.59), Pulikottil Shaju et al..$^{10}$ en India (RM = 2.94; IC $95 \%$ 1.9-4.6) y Mega et al. ${ }^{22}$ en Kosovo $(\mathrm{RM}=3.4 ; \mathrm{IC} 95 \%$ 1.6-7.9). Además, en Chile, López et al. ${ }^{23}$ en un ensayo clínico demostraron que la EP es factor de riesgo para NP $(\mathrm{RM}=4.70$; IC $95 \%$ 1.29-17.13).
Por otra parte, en diferentes investigaciones no se ha encontrado asociación entre EP y mayor frecuencia de NP, como las efectuadas por Wood et al. en Canadá, ${ }^{11}$ Moore et al. en Reino Unido ${ }^{24}$ y Lohsoonthom et al. en Tailandia $^{12}$ en diseños de casos y controles, así como Calabrese et al. ${ }^{13}$ en Italia en un estudio transversal.

En la mayoría de los estudios revisados, ${ }^{6,8-13}$ el diagnóstico de EP en las madres se llevó a cabo poco después del nacimiento del RN, de la misma manera como se llevó a cabo en la presente investigación, y se emplearon criterios diagnósticos basados en los signos clínicos de inflamación (tener dientes con al menos un sitio con profundidad $\geq 4 \mathrm{~mm}$ y pérdida clínica de tejido óseo o blando $\geq 3 \mathrm{~mm}),{ }^{15}$ semejante a lo reportados por otros investigadores. ${ }^{6-11,13}$ También, la fuerza de la asociación entre EP y NP encontrada en este estudio (RM $=2.25$, IC $95 \%=1.61$ 3.14) fue consistente con la reportada por Ofenbacher et al., ${ }^{8}$ Siqueira et al. ${ }^{9}$ y Pulikottil Shaju Jacob et al. ${ }^{10}$ En una revisión sistemática realizada por Xiong et al. ${ }^{5}$ acerca de la EP y desenlaces adversos en el embarazo se sugiere que estos pueden ser peores en niveles socioeconómicos bajos y con pobre acceso al cuidado de la salud dental, como ocurrió en la presente investigación, en la que $89 \%$ de las mujeres de los casos y $76 \%$ de los controles nunca tuvieron revisión dental.

También, Xiong et al. ${ }^{5}$ y Lohsoonthom et al. ${ }^{12}$ mencionan que estudios llevados a cabo en Europa, donde los ciudadanos tienen salud dental universal, no han encontrado asociación entre EP y NP. 
La documentación del papel de la EP como factor de riesgo independiente para NP es importante debido que existen pocas publicaciones que han estudiado esta relación en América Latina.

$\mathrm{Zi}$ et al. ${ }^{25}$ mencionan que se ha propuesto que la EP se relaciona con NP debido a que los microorganismos y sus productos procedentes de los sitios subgingivales pueden llegar al liquido amniótico por vía hematógena y dar inicio a la vía proinflamatoria en la placenta y los tejidos fetales; además, los patógenos de los tejidos gingivales contribuyen a la liberación de mediadores adicionales que se diseminan en forma sistémica y de esta forma se crea un ambiente inflamatorio que activa el inicio de la labor y restringe el crecimiento del feto.

Una limitación de este estudio fue que no se determinó la edad gestacional con ultrasonidos seriados en todos los embarazos, en parte debido a la naturaleza retrospectiva del diseño de investigación; para aumentar la precisión de la valoración de la edad gestacional por FUM, esta se corrigió con los métodos de Capurro y Ballard. Cabe comentar que el diseño de casos y controles no permite documentar asociación causal, sin embargo, la demostración de que la EP fue un factor de riesgo independiente para NP permite emprender medidas preventivas, sobre todo en grupos vulnerables como las mujeres en edad reproductiva con escaso acceso al cuidado dental.

Por todo lo anterior, el presente estudio permite concluir que la EP es un factor de riesgo prevenible para NP y refuerza la importancia de su prevención, detección y tratamiento antes y durante el embarazo.

\section{Bibliografía}

1. Ananth CV, Vintzileos AM. Epidemiology of preterm birth and its clinical subtypes. J Matern Fetal Neonatal Med. 2006;19:773-782.

2. Purish SE, Gyamfi-Bannerman C. Epidemiology of preterm birth. Semin Perinatol. 2017:41:387-393.

3. Goldenberg RL, Culhane JF, lams JD, Romero R. Epidemiology and causes of preterm birth. Lancet. 2008;371:75-84.

4. Pérez-Molina J, Quezada-López C, Panduro-Barón G, Castro-Hernández JF. Factores de riesgo materno asociados con muerte fetal en nacimientos pretérmino en un hospital público del occidente de México. Rev Invest Clin. 2012;64:330-335.
5. Xiong X, Bueckens P, Fraser WD, Beck J, Offenbacher S. Periodontal disease and adverse pregnancy outcomes: a systematic review. BJOG. 2006;113:135-143.

6. Offenbacher S, Katz V, Fertik G, Collins J, Boyd D, Maynor G, et al. Periodontal infection as a possible risk factor for preterm low birth weight. J Periodontol. 1996;67:1103-1113.

7. Jeffcoat MK, Geurs NC, Reddy MS, Cliver SP, Goldenberg RL, Hauth JC. Periodontal infection and preterm birth: results of a prospective study. J Am Dent Assoc. 2001;132:875-880.

8. Ofenbacher S, Boggess KA, Murtha AP, Jared HL, Lieff S, McKaig RG, et al. Progressive periodontal disease and risks of very preterm delivery. Obstet Gynecol. 2006;107:29-36.

9. Siqueira FM, Cota LO, Costa JE, Haddad JP, Lana AM, Costa FO. Intrauterine growth restriction, low birth weight, and preterm birth: adverse pregnancy outcomes and their association with maternal periodontitis. J Periodontol. 2007;78:2266-2276.

10. Pulikottil-Shaju J, Nath S. Periodontitis among poor rural Indian mothers increases the risk of low birth weight babies: a hospital-based case control study. J Periodontal Implant Sci. 2014;44:85-93.

11. Wood S, Frydman A, Cox S, Brant R, Needoba S, Eley B, et al. Periodontal disease and spontaneous preterm birth: case control study. BMC Pregnancy Childbirth. 2006;6:24.

12. Lohsoonthron V, Kungsadalpipob K, Chanchareonsook P, Limpongsanurak $S$, Vanichjakvong $O$, Sutdhibhisal $S$, et al. Is maternal periodontal disease a risk factor for preterm birth? Am J Epidemiol. 2009; 169:731-739.

13. Calabrese N, Calabrese A, Nibali L, Rosati A, Fiengo S, Di-Renzo GC. Is there any association between periodontitis and preterm low birth weight? J Matern Fetal Neonatal Med. 2010;23:1288-1293.

14. Newman TB, Browner WS, Cummings SR, Hulley SB. Designing an observational study: cross-sectional and case-control studies. En: Hulley SB, Cummings SR, Browner WS, Grady D, Hearst N, Newman TB, editores. Designing clinical research an epidemiologic approach. EE. UU.: Lippincott Williams \& Wilkins; 2001.

15. Highfield J. Diagnosis and classification of periodontal disease. Aust Dent J. 2009;54:S11-S26.

16. Capurro H, Konichezky S, Fonseca D, Caldeyro-Barcia R. A simplified method for diagnosis of gestational age in the newborn infant. J Pediatr. 1978;93:120-122.

17. Ballard JL, Khoury JC, Wedif K, Wang L, Eilers-Walsman BL, Lipp R. New Ballard score, expanded to include extremely premature infants. J Pediatr. 1991;119:417-423.

18. Cerda J, Villarroel L. Evaluación de la concordancia inter-observador en investigación pediátrica: coeficiente de Kappa. Rev Chil Pediatr. 2008;79:54-58.

19. Norma Oficial Mexicana NOM-007-SSA2-1993, atención de la mujer durante el embarazo, parto y puerperio y del recién nacido. Criterios y procedimientos para la prestación del servicio. México: Diario Oficial de la Federación 1994 Oct 31.

20. Adimark. El nivel socio económico Esomar. Manual de Aplicación. Chile; 2000.

21. Pérez-Molina JJ, Cobián-López B, Silva-Maciel CA. Factores de riesgo materno y nacimiento pretérmino en un hospital público del occidente de México. Ginecol Obstet Mex. 2004;72:142-149.

22. Meqa K, Dragidella F, Disha M, Sllamniku-Dalipi Z. The association between periodontal disease and preterm low birthweight in Kosovo. Acta Stomatol Croat. 2017;51:33-40.

23. López NJ, Smith PC, Gutierrez J. Periodontal therapy may reduce the risk of preterm low birth weight in women with periodontal disease: a randomized controlled trial. J Periodontol. 2002;73:911-924

24. Moore S, Randhawa M, Ide M. A case-control study to investigate an association between adverse pregnancy outcome and periodontal disease. J Clin Periodontol. 2005;32:1-5.

25. Zi MY, Longo PL, Bueno-Silva B, Mayer MP. Mechanisms involved in the association between periodontitis and complications in pregnancy. Front Public Health. 2015;29:2:290. 